\title{
A relevância de fármacos antidepressivos para o tratamento de disfunções musculares faciais crônicas
}

\author{
The relevance of antidepressant drugs for the treatment of chronic muscular dysfunctions \\ facial
}

\author{
La relevancia de las drogas antidepresivas para el tratamiento de las disfunciones \\ musculares facial crónicas
}

\begin{abstract}
Samara Kelly da Silva Cavalcante ${ }^{1}$, Maria Priscylliana de Fátima Arcelino Couto ${ }^{1}$, Talita Arrais Daniel Mendes $^{1,2 *}$, Lucas Lino de Oliveira ${ }^{1}$, Teófilo Felipe Santiago ${ }^{1}$, Ana Carolina Matias Dinelly Pinto ${ }^{1}$, Raynara de Sousa Brito ${ }^{1}$, Tarlóvia Cavalcante Noronha1 ${ }^{1}$, Vilana Maria Adriano Araújo², Érika Matias Pinto Dinelly ${ }^{1}$.
\end{abstract}

\section{RESUMO}

Objetivo: Revisar a literatura acerca da eficácia de fármacos antidepressivos no tratamento de pacientes com disfunções musculares faciais crônicas em termos de redução da dor e efeitos adversos. Métodos: Pesquisaram-se os descritores "Antidepressive Agents", "Facial Pain", "Drug Therapy,", conectados pelo operador booleano "AND", na base de dados Pubmed dos últimos 10 anos. Obtiveram-se 40 artigos, dos quais foram selecionados $10 \mathrm{com}$ base na leitura de títulos e resumos. Foram incluídos estudos e ensaios clínicos que relataram tratamentos de disfunções musculares faciais crônicas com fármacos antidepressivos. Resultados: 3 estudos concluíram que houve redução da dor em pacientes tratados com Amitriptilina e naqueles que receberam Amitriptilina com Pindolol. 2 estudos perceberam efeitos positivos nos pacientes tratados com Milnacipran. 2 estudos inferiram que, após o início da administração de Duloxetina, houve redução significativa da dor. 2 estudos sugeriram eficácia em pacientes com disfunções musculares faciais crônicas e que utilizaram Amitriptilina e Nortriptilina. 1 estudo observou o uso de Tizanidina ou Ciclobenzaprina, e concluiu sua ineficácia comparado ao placebo. Considerações finais: Os fármacos antidepressivos possuem a capacidade de tratar com eficácia as dores miofasciais, principalmente dores crônicas, devido a capacidade de possuir efeitos analgésicos, sendo a Amitriptilina considerada padrão-ouro para esses tratamentos.

Palavras-chave: Fármacos antidepressivos, Dor facial, Terapia combinada.

\section{ABSTRACT}

Objective: To review the literature on the efficacy of antidepressant drugs in the treatment of patients with chronic facial muscle disorders in terms of pain reduction and adverse effects. Methods: The descriptors "Antidepressive Agents", "Facial Pain", "Drug Therapy,", connected by the Boolean operator "AND", were searched in the Pubmed database of the last 10 years. Forty articles were obtained, of which 10 were selected based on the reading of titles and abstracts. Studies and clinical trials that reported treatments of chronic facial muscle dysfunction with antidepressant drugs were included. Results: 3 studies concluded that there was a reduction in pain in patients treated with Amitriptyline and in those who received Amitriptyline with Pindolol. 2 studies have found positive effects in patients treated with Milnacipran. 2 studies inferred that, after the start of Duloxetine administration, there was a significant reduction in pain. 2 studies suggested efficacy in patients with chronic facial muscle dysfunction and who used Amitriptyline and Nortriptyline. 1 study observed the use of Tizanidine or Cyclobenzaprine, and concluded its ineffectiveness compared to placebo. Final considerations: Antidepressant drugs have the ability to effectively treat myofascial pain, especially chronic pain, due to the ability to have analgesic effects, with Amitriptyline being considered the gold standard for these treatments.

Key words: Antidepressant drugs, Facial pain, Combination therapy.

${ }^{1}$ Centro Universitário Católica de Quixadá (Unicatólica), Quixadá - CE. *E-mail: talita_arrais@hotmail.com

2 Universidade Federal do Ceará (UFC), Fortaleza - CE. 


\section{RESUMEN}

Objetivo: Revisar la literatura sobre la efectividad de los fármacos antidepresivos en el tratamiento de pacientes con disfunción crónica de los músculos faciales en términos de reducción del dolor y efectos adversos. Métodos: Se buscaron en la base de datos Pubmed de los últimos 10 años los descriptores "Agentes antidepresivos", "Dolor facial", "Terapia farmacológica", conectados por el operador booleano "AND". Se obtuvieron cuarenta artículos, de los cuales se seleccionaron 10 en base a la lectura de títulos y resúmenes. Se incluyeron estudios y ensayos clínicos que informaron tratamientos de la disfunción crónica de los músculos faciales con fármacos antidepresivos. Resultados: 3 estudios concluyeron que hubo una reducción del dolor en los pacientes tratados con Amitriptilina y en los que recibieron Amitriptilina con Pindolol. 2 estudios han encontrado efectos positivos en pacientes tratados con milnacipran. 2 estudios infirieron que, tras el inicio de la administración de duloxetina, hubo una reducción significativa del dolor. 2 estudios sugirieron eficacia en pacientes con disfunción crónica de los músculos faciales y que usaban Amitriptilina y Nortriptilina. Un estudio observó el uso de tizanidina o ciclobenzaprina y concluyó su ineficacia en comparación con placebo. Consideraciones finales: Los medicamentos antidepresivos tienen la capacidad de tratar eficazmente el dolor miofascial, especialmente el dolor crónico, debido a la capacidad de tener efectos analgésicos, y la amitriptilina se considera el estándar de oro para estos tratamientos.

Palabras clave: Fármacos antidepresivos, Dolor facial, Terapia combinada.

\section{INTRODUÇÃO}

A dor orofacial é um termo amplo que compreende múltiplas condições dolorosas que afetam a cavidade oral e a face. Dentro dessa, a síndrome da dor miofascial (SDM) é a segunda mais recorrente e está frequentemente associada à disfunção temporomandibular (DTM). A SDM surge da dor musculoesquelética aguda e crônica e, normalmente, tem um componente neuropático referido. $O$ diagnóstico é dado por meio da presença de pontos-gatilho miofasciais (PGMs) localizados em nós contraídos associados a fibras musculares tensas; essas fibras musculares tensas se estendem ao apego muscular chamado bandas esticadas. Os PGMs, geralmente, encontram-se rígidos e provocam dor ao serem palpados.

No exame microscópico são observadas fibras musculares hipercontraídas associadas à liberação sustentada de cálcio no retículo sarcoplasmático, causada pela geração contínua de potencial de ação devido à intensa atividade neural (VAROLI FK, et al., 2015; CONNELLY, et al., 2016; ABBOUD WA, et al., 2017).

A dor miofascial está localizada unilateralmente, principalmente no ângulo da mandíbula e na área préauricular, apresentando sintomatologia dolorosa de intensidade moderada e de qualidade insensível, que tende a aumentar com a função. A etiologia da SDM é entendida por meio de um modelo biopsicossocial que reflete em uma interação complexa entre fatores físicos, comportamentais, sociais e psicológicos. Por sua vez, o tratamento das disfunções musculares é, na maioria dos casos, multidisciplinar, na qual a terapia reversível conservadora é a estratégia preferida. As opções de tratamento primário incluem fisioterapia, dieta leve, eliminação de hábitos parafuncionais e terapia com gelo úmido. A terapia com placa oclusal é um complemento útil para pacientes com SDM. No entanto, a dor miofascial pode se tornar crônica e persistente em cerca de um terço dos pacientes, e o tratamento farmacoterapêutico a longo prazo é frequentemente um componente essencial de manejo nesses casos (NAGASHIMA W, et al., 2012; KRONER JW, et al., 2017; DALEWSKI B, et al., 2019).

Além da multidisciplinariedade que se faz necessária para o tratamento, existem terapias que vêm apresentando efeitos positivos em relação a dor miofascial, entre elas, o uso do ibuprofeno da classe dos anti-inflamatórios não esteroidais; a pioglitazona do grupo das tiazolidinedionas; diazepam da classe dos benzodiazepínicos. Atualmente, os antidepressivos tricíclicos (ADT) vem em forma de terapia apresentando eficácia e rapidez no tratamento sem acarretar ao paciente a presença de tantos efeitos adversos (ALVES ACM, et al., 2012; LYONS DN, et al., 2018; NAGAKURA Y, et al., 2019).

Os antidepressivos tricíclicos (ADT) estão entre as opções de tratamento farmacológico mais aceitas. A Amitriptilina (AMT) é o ADT mais estudado e é frequentemente o medicamento de escolha para o tratamento da dor miofascial persistente (MFP), com doses variando de 10 a $35 \mathrm{mg}$ por dia. Os ADT são metabolizados no fígado e seus efeitos analgésicos são mediados principalmente por uma inibição central da recaptação de serotonina (5-HT) e noradrenalina. No entanto, outros modos de ação incluem níveis aumentados de opioides 
cerebrais endógenos, efeitos antagonistas do N-metil-D-aspartato (NMDA), bloqueio dos canais de sódio, cálcio e potássio, regulação positiva da expressão do receptor GABA e histamina, além do bloqueio do receptor colinérgico (KIMURA H, et al., 2012; HAVIV Y, et al., 2015; URQUHART DM, et al., 2018).

O efeito analgésico de um ADT na dor crônica é independente de sua ação antidepressiva, e aparece em doses significativamente mais baixas do que as necessárias para a depressão (75 a $150 \mathrm{mg} / \mathrm{dia}$ ). Os efeitos adversos (EA) são comuns, mas geralmente leves, e a administração crônica de $25 \mathrm{mg}$ de AMT diariamente não está associada a reduções significativas no processamento do paciente ou na capacidade de execução de tarefas. Os EAs incluem sedação, palpitação, náusea, boca seca, prisão de ventre, tontura, cansaço e fadiga, além de ganho de peso devido ao aumento do apetite (BROWN S, et al., 2016; KOBAYASHI Y, et al., 2017; HAVIV Y, et al., 2018).

Diante disso, o objetivo deste trabalho é revisar a literatura acerca da eficácia de fármacos antidepressivos no tratamento de pacientes com disfunções musculares faciais crônicas em termos de redução da dor e efeitos adversos.

\section{MÉTODOS}

Para esta revisão de literatura, foi realizada uma busca eletrônica na base de dados Sistema Online de Busca e Análise de Literatura Médica (PubMed), utilizando os seguintes descritores em inglês: Antidepressive Agents, Facial Pain e Drug Therapy, cadastrados nos descritores em ciência da saúde (Decs), sendo "AND" o operador Booleano. Após a delimitação de 10 anos para a busca, foram encontrados 40 artigos.

Com base na leitura de títulos e resumos, foram incluídos estudos e ensaios clínicos que relataram tratamentos de disfunções musculares crônicas com fármacos antidepressivos publicados no período de 2010 a 2020, com disponibilidade integral do texto e clareza no detalhamento metodológico. Foram excluídos artigos que não apresentaram relevância clínica sobre o tema abordado, revisões narrativas de literatura, monografias, além de estudos com metodologia incompletas e resultados inconclusivos. Após a leitura crítica de títulos e resumos, selecionou-se 10 artigos.

Figura 1 - Fluxograma para a busca de artigos científicos

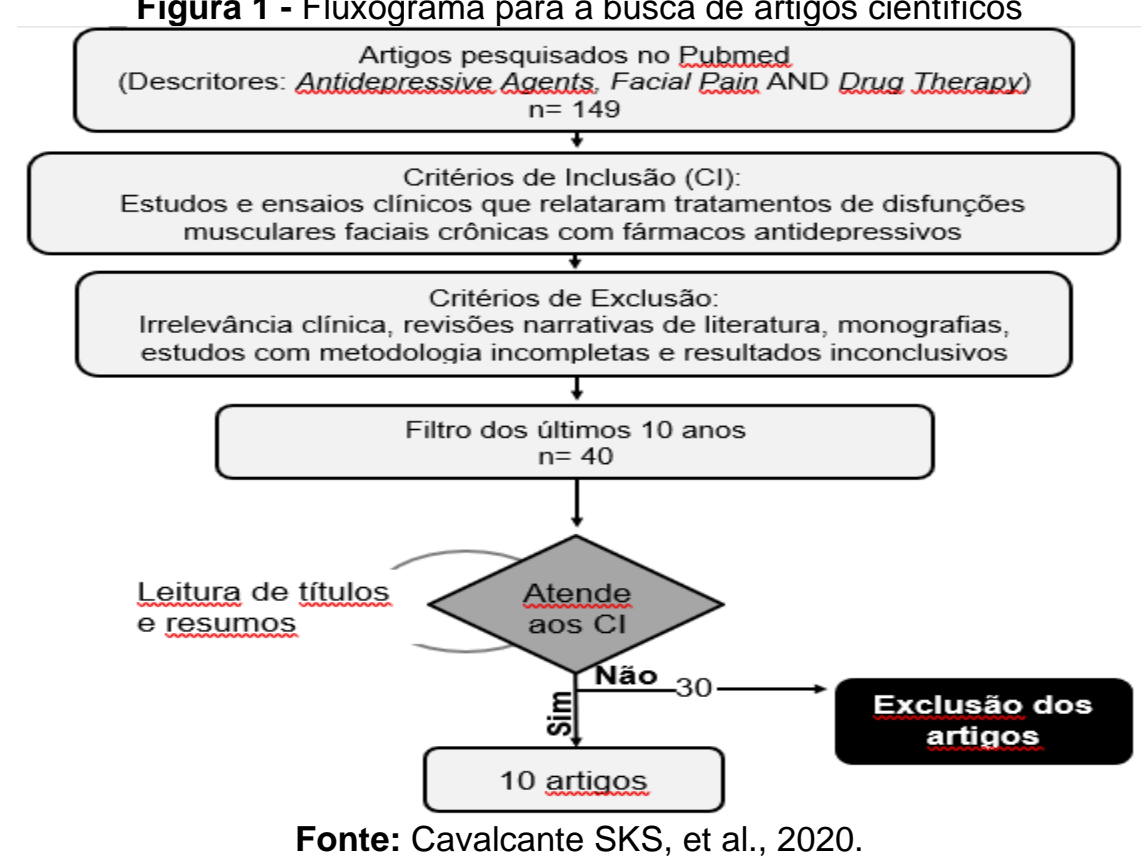

\section{RESULTADOS}

Após a busca por artigos que explicassem os assuntos em diferentes aspectos, procurou-se nesta revisão de literatura juntá-los em uma tabela, a fim de melhorar o entendimento dos resultados alcançados. Obtiveram-se 9 ensaios clínicos e 1 estudo de coorte. Os estudos confirmaram a relevância do uso de fármacos antidepressivos para o tratamento de disfunções musculares crônicas (Quadro 1). 
Quadro 1 - Estudos que avaliaram a eficácia de fármacos antidepressivos para o tratamento de disfunções musculares faciais crônicas.

\begin{tabular}{|c|c|c|c|}
\hline Autor/Ano & Título & Metodologia & Resultados \\
\hline $\begin{array}{l}\text { ITO M, et } \\
\text { al., } 2010\end{array}$ & $\begin{array}{l}\text { Effectiveness of Milnacipran } \\
\text { for the Treatment of Chronic } \\
\text { Pain in the Orofacial Region }\end{array}$ & $\begin{array}{l}\text { Milnacipran foi administrado por } 12 \text { semanas a } 36 \text { pacientes com dor } \\
\text { crônica na região orofacial ( }(3 \text { homens e } 29 \text { mulheres, com idade entre } \\
22 \text { e } 76 \text { anos e média de idade de } 59 \text { anos). Desses pacientes, } 22 \text { e } 10 \\
\text { pacientes tinham SBA e OA, respectivamente. A dose inicial de } \\
\text { Milnacipran foi de } 15 \text { mg por dia e a dose foi aumentada para } 100 \mathrm{mg} \\
\text { por dia. }\end{array}$ & $\begin{array}{l}\text { A dor diminuiu significativamente após o tratamento de } 12 \\
\text { semanas e mostrou um curso de declínio semelhante, } \\
\text { independentemente dos sintomas depressivos } \\
\text { simultâneos durante as } 12 \text { semanas. }\end{array}$ \\
\hline $\begin{array}{l}\text { KIMURA H, } \\
\text { et al., } 2012\end{array}$ & $\begin{array}{l}\text { Plasma levels of milnacipran } \\
\text { and its effectiveness for the } \\
\text { treatment of chronic pain in } \\
\text { the orofacial region }\end{array}$ & $\begin{array}{l}\text { Um total de } 44 \text { pacientes tomou Milnacipran por } 12 \text { semanas. Os } \\
\text { pacientes foram avaliados quanto à dor e sintomas depressivos usando } \\
\text { a escala visual analógica (EVA) e a escala de avaliação de depressão } \\
\text { de Hamilton, respectivamente. O nível de Milnaciprano plasmático } \\
\text { também foi avaliado na semana } 12 \text {. }\end{array}$ & $\begin{array}{l}\text { Não foi observada relação significativa entre a } \\
\text { porcentagem de diminuição no escore da Hamilton } \\
\text { Depression Rating Scale e o nível de Milnaciprano no } \\
\text { plasma. }\end{array}$ \\
\hline $\begin{array}{l}\text { NAGASHI } \\
\text { MA W, et } \\
\text { al., } 2012\end{array}$ & $\begin{array}{l}\text { Effectiveness of Duloxetine } \\
\text { for the Treatment of Chronic } \\
\text { Nonorganic Orofacial Pain }\end{array}$ & $\begin{array}{l}\text { Neste estudo de } 12 \text { semanas, a Duloxetina foi administrada em uma } \\
\text { dose flexível fixa de } 20 \text { a } 40 \mathrm{mg} / \mathrm{d} \text { a } 41 \text { pacientes com síndrome da boca } \\
\text { ardente e/ou odontalgia atípica. A dor foi avaliada usando a } \\
\text { EVA sendo } 12 \text { semanas de tratamento. Os sintomas depressivos foram } \\
\text { avaliados usando a Hamilton Depression Rating Scale na linha de base } \\
\text { e às } 12 \text { semanas de tratamento. }\end{array}$ & $\begin{array}{l}\text { Redução de dor a partir de } 2 \text { semanas de tratamento em } \\
\text { comparação com a pontuação basal. }\end{array}$ \\
\hline $\begin{array}{l}\text { AGIUS AM, } \\
\text { et al., } 2013\end{array}$ & $\begin{array}{l}\text { A Randomized Controlled } \\
\text { Trial comparing the efficacy } \\
\text { of low-dose amitriptyline, } \\
\text { amitriptyline with pindolol and } \\
\text { surrogate placebo in the } \\
\text { treatment of chronic tension- } \\
\text { type facial pain }\end{array}$ & $\begin{array}{l}\text { Sessenta e dois pacientes foram randomizados para três grupos de } \\
\text { tratamento: (a) AMT } 10 \mathrm{mg} \text { por dia, (b) AMT } 10 \mathrm{mg} \text { por dia com Pindolol } \\
5 \mathrm{mg} \text { duas vezes ao dia e (c) Loratadina } 10 \mathrm{mg} \text { por dia. Os escores } \\
\text { diários de dor usando um diário de dor facial foram registrados durante } \\
\text { oito semanas. }\end{array}$ & $\begin{array}{l}\text { Em } 8 \text { semanas, a frequência e intensidade da dor foram } \\
\text { significativamente reduzidas nos pacientes tratados com } \\
\text { AMT e naqueles que receberam AMT com Pindolol em } \\
\text { comparação ao placebo substituto. }\end{array}$ \\
\hline $\begin{array}{l}\text { AGIUS AM, } \\
\text { et al., } 2013\end{array}$ & $\begin{array}{l}\text { Serial blood serotonin levels } \\
\text { in a randomized controlled } \\
\text { trial comparing the efficacy of } \\
\text { low-dose amitriptyline, } \\
\text { amitriptyline with pindolol and } \\
\text { surrogate placebo in patients } \\
\text { with chronic tension-type } \\
\text { facial pain }\end{array}$ & $\begin{array}{l}\text { Sessenta e dois pacientes foram randomizados para três grupos de } \\
\text { tratamento a) AMT, b) AMT com Pindolol e c) Loratadina como placebo } \\
\text { substituto. A serotonina no sangue total foi tomada antes e após } 8 \\
\text { semanas de tratamento. A serotonina também foi medida em } 40 \\
\text { controles saudáveis pareados pela idade. }\end{array}$ & $\begin{array}{l}\text { Houve uma redução significativa nos níveis de serotonina } \\
\text { no sangue na AMT com o grupo Pindolol, sem alteração } \\
\text { nos níveis de serotonina no grupo placebo substituto. As } \\
\text { mulheres do grupo controle apresentaram níveis } \\
\text { significativamente mais altos de serotonina do que os } \\
\text { homens. Mulheres com dor facial do tipo tensão que não } \\
\text { responderam ao tratamento apresentaram serotonina no } \\
\text { sangue significativamente menor do que as mulheres do } \\
\text { grupo controle. }\end{array}$ \\
\hline
\end{tabular}

REAS/EJCH | Vol.12(10) | e4729 | DOI: https://doi.org/10.25248/reas.e4729.2020 Página $\mathbf{4}$ de $\mathbf{1 0}$ 


\begin{tabular}{|c|c|c|c|}
\hline Autor/Ano & Título & Metodologia & Resultados \\
\hline $\begin{array}{l}\text { AGIUS AM, } \\
\text { et al., } 2014\end{array}$ & $\begin{array}{l}\text { Prospective three-year follow } \\
\text { up of a cohort study of } 240 \\
\text { patients with chronic facial } \\
\text { pain }\end{array}$ & $\begin{array}{l}\text { Uma corte de } 240 \text { pacientes com dor facial crônica foi acompanhada por } \\
36 \text { meses em uma clínica otorrinolaringológica em Malta. Os tipos de } \\
\text { dor facial foram classificados de acordo com os critérios da } \\
\text { Classificação Internacional de Dor de Cabeça. O índice de massa } \\
\text { corporal, ocupação e nível educacional dos pacientes foram } \\
\text { comparados com a população geral. }\end{array}$ & $\begin{array}{l}\text { Dor no meio da face do tipo tensão e enxaqueca facial } \\
\text { sem aura foram os tipos mais comuns de dor facial } \\
\text { crônica. Após três anos, quase metade dos pacientes não } \\
\text { apresentava sintomas e, em um terço, a dor passou de } \\
\text { crônica para episódica. }\end{array}$ \\
\hline $\begin{array}{l}\text { ALENCAR } \\
\text { F, et al., } \\
2014\end{array}$ & $\begin{array}{l}\text { Patient Education and Self- } \\
\text { Care for the Management of } \\
\text { Jaw Pain upon Awakening: a } \\
\text { randomized controlled } \\
\text { clinical trial comparing the } \\
\text { effectiveness of adding } \\
\text { pharmacologic treatment with } \\
\text { cyclobenzaprine or tizanidine }\end{array}$ & $\begin{array}{l}\text { Quarenta e cinco pacientes com diagnóstico de dor miofascial. Os } \\
\text { sujeitos foram divididos aleatoriamente em um dos três grupos: grupo } \\
\text { placebo, grupo TZA (4 mg) ou grupo CIC (10 mg). Os pacientes foram } \\
\text { avaliados quanto a alterações na intensidade, frequência e duração da } \\
\text { dor, usando o Índice de Sintomas de Gravidade modificado e alterações } \\
\text { na qualidade do sono com o uso do Índice de Qualidade do Sono de } \\
\text { Pittsburgh. }\end{array}$ & $\begin{array}{l}\text { Todos os grupos apresentaram redução nos sintomas de } \\
\text { dor e melhora na qualidade do sono. No entanto, não } \\
\text { foram observadas diferenças significativas entre os } \\
\text { grupos na avaliação pós-tratamento. }\end{array}$ \\
\hline $\begin{array}{l}\text { HAVIV } \\
\text { Y, et al., } \\
2015\end{array}$ & $\begin{array}{l}\text { Myofascial Pain: an open } \\
\text { study on the } \\
\text { pharmacotherapeutic } \\
\text { response to stepped } \\
\text { treatment with tricyclic } \\
\text { antidepressants and } \\
\text { gabapentin }\end{array}$ & $\begin{array}{l}\text { Foi utilizado um protocolo farmacoterapêutico escalonado. Todos os } 42 \\
\text { pacientes com dor MFP com sensibilidade dos músculos regionais } \\
\text { receberam primeiro AMT, mas aqueles com efeitos colaterais foram } \\
\text { posteriormente transferidos para Nortriptilina (NOR). Nos pacientes em } \\
\text { que não foi observada resposta aos ADT, iniciou-se a Gabapentina. }\end{array}$ & $\begin{array}{l}\text { Nos pacientes tratados com ADT, } 43 \% \text { apresentaram } \\
\text { redução } \geq 50 \% \text { na intensidade da dor. Isto foi alcançado } \\
\text { com uma dose média de AMT de } 16 \pm 1,1 \mathrm{mg} / \mathrm{d} \text { e uma } \\
\text { dose média de NOR de } 25 \pm 2,1 \mathrm{mg} / \mathrm{d} \text {. Os pacientes que } \\
\text { não responderam aos ADTs foram caracterizados por } \\
\text { uma idade significativamente mais alta, doenças médicas } \\
\text { mais comórbidas e evidências de maior disseminação da } \\
\text { dor regional ( } P<0,05) \text {. Apesar de não responderem aos } \\
\text { ADTs, } 36,8 \% \text { desse grupo apresentaram redução } \geq 50 \% \\
\text { na intensidade da dor após a terapia com Gabapentina } \\
\text { em uma dose diária média de } 973,7 \pm 68,8 \text { mg. }\end{array}$ \\
\hline $\begin{array}{l}\text { KOBAYAS } \\
\text { HI Y, et al., } \\
\quad 2017\end{array}$ & $\begin{array}{l}\text { Duloxetine Plasma } \\
\text { Concentrations and Its } \\
\text { Effectiveness in the } \\
\text { Treatment of Nonorganic } \\
\text { Chronic Pain in the Orofacial } \\
\text { Region }\end{array}$ & $\begin{array}{l}\text { Foram administrados Duloxetina em } 77 \text { pacientes diagnosticados como } \\
\text { portadores de síndrome da boca ardente ou odontalgia atípica por } 12 \\
\text { semanas. A dose inicial de Duloxetina foi estabelecida em } 20 \mathrm{mg} / \mathrm{d} \text { e } \\
\text { aumentada para } 40 \mathrm{mg} \text { / d após uma semana. A dor foi avaliada } \\
\text { utilizando a EVA e os sintomas depressivos usando o Structured } \\
\text { Interview Guide for the Hamilton Depression Rating Scale e mediram as } \\
\text { concentrações plasmáticas de Duloxetina } 12 \text { semanas após o início de } \\
\text { sua administração. }\end{array}$ & $\begin{array}{l}\text { A dor foi significativamente menor } 12 \text { semanas após o } \\
\text { início da administração de Duloxetina. }\end{array}$ \\
\hline $\begin{array}{l}\text { HAVIV } \\
\text { Y, et al., } \\
2019\end{array}$ & $\begin{array}{l}\text { Nortriptyline Compared to } \\
\text { Amitriptyline for the } \\
\text { Treatment of Persistent } \\
\text { Masticatory Myofascial Pain }\end{array}$ & $\begin{array}{l}\text { Cinquenta pacientes com MFP crônica foram incluídos no estudo; } 30 \\
\text { foram medicados apenas com AMT e } 20 \text { tomaram NOR após } \\
\text { descontinuar o AMT devido a efeitos adversos. }\end{array}$ & $\begin{array}{l}\text { Os pacientes com NOR relataram um EVD final menor } \\
\text { em comparação aos pacientes com AMT. A taxa de } \\
\text { melhora de } 50 \% \text { com o tratamento NOR foi melhor do que } \\
\text { com o tratamento com AMT }(P=0,036) \text {. }\end{array}$ \\
\hline
\end{tabular}

Legenda: SBA: Síndrome da Boca Ardente, OA: Odontalgia Atípica, EVA: Escala Visual Analógica, AMT: Amitriptilina MFP: Dor Miofascial Persistente, TZA: Tizanidina, CIC: Ciclobenzaprina, ADT: Antidepressivos Tricíclicos, NOR: Nortriptilina. Fonte: Cavalcante SKS, et al., 2020.

REAS/EJCH | Vol.12(10) | e4729 | DOI: https://doi.org/10.25248/reas.e4729.2020 Página $\mathbf{5}$ de $\mathbf{1 0}$ 


\section{DISCUSSÃO}

Pacientes que sofrem da SDM possuem quadros clínicos de diferentes formas, desde quadros leves até quadros mais crônicos e complexos, devido a isso inúmeros tratamentos são idealizados visando a terapia que desencadeie a melhor resposta. Com isso, o processo de reabilitação geralmente é prolongado e dependente da educação e da responsabilidade do paciente, além do desenvolvimento de uma parceria entre fisioterapeuta-paciente, baseada na confiança mútua. Em longo prazo, a conduta não reside apenas no tratamento dos pontos-gatilhos, mas na identificação e modificação dos fatores contribuintes, visto que estes estão relacionados aos aspectos biopsicossociais dos pacientes (BATISTA SJ, et al., 2012).

Em decorrência disso, faz-se necessária uma abordagem terapêutica interdisciplinar composta por vários especialistas (fisioterapeutas, cirurgiões-dentistas, psicólogos e psiquiatras), com o intuito de proporcionar tratamentos eficazes. Dentre eles existem os tratamentos fisioterápicos; acupuntura; técnicas de agulhamento; suporte psicológico; identificação de fatores que desencadeiam a condição, bem como o tratamento fazendo uso de fármacos. Dentre essa ampla classe destacam-se os antidepressivos tricíclicos (ADT) (CONNELLY ST, et al., 2016).

Os ADTs são aprovados pela Food and Drug Administration (FDA) para tratar uma variedade de doenças, dependendo da formulação. Os ADTs que têm aprovação da FDA para tratar transtornos depressivos incluem Amitriptilina, Amoxapina, Doxepina, Desipramina, Nortriptilina, Protriptilina, Imipramina e Trimipramina. Em termos de tratamento do transtorno depressivo maior, os ADTs mostram eficácia equívoca com inibidores seletivos da recaptação de serotonina, porém os ADTs causam efeitos adversos mais significativos devido à sua atividade anticolinérgica e um limiar mais baixo para overdose (BOAFO A, et al., 2020).

Por esses motivos, esses medicamentos geralmente não são usados como tratamento de primeira linha do transtorno depressivo maior. Os usos off-label (não-FDA) dos ADTs incluem profilaxia da enxaqueca, transtorno obsessivo-compulsivo, insônia, ansiedade e dor crônica, especialmente condições de dor neuropáticas, como dor miofascial, neuropatia diabética e neuralgia pós-herpética. Especificamente, para a profilaxia da enxaqueca e dor miofascial, a Amitriptilina é mais comumente usada (XU XM, et al., 2017)

Os antidepressivos tricíclicos atuam em aproximadamente cinco vias diferentes de neurotransmissores para alcançar seus efeitos. Eles bloqueiam a recaptação de serotonina e noradrenalina nos terminais présinápticos, o que leva ao aumento da concentração desses neurotransmissores na fenda sináptica. $O$ aumento da concentração de serotonina na sinapse provavelmente contribui para seu efeito antidepressivo. Além disso, eles atuam como antagonistas competitivos nos receptores alfa colinérgicos pós-sinápticos (alfa1 e alfa2), muscarínicos e histaminérgicos (GARROTE CEM, et al., 2010).

A estrutura química de um ADT consiste em uma estrutura de três anéis com uma amina secundária ou terciária ligada. As aminas secundárias incluem Desipramina, Nortriptilina e Protriptilina, enquanto as aminas terciárias consistem em Amitriptilina, Clomipramina, Doxepina, Imipramina e Trimipramina. As aminas terciárias tendem a ter maior bloqueio da recaptação de serotonina, enquanto as aminas secundárias têm maior bloqueio da captação de noradrenalina. A combinação de diferentes estruturas aminas e variações na composição química contribuem para a multiplicidade de efeitos adversos observados com o uso de ADT, pois esses fatores afetam a afinidade e ligação do receptor de ADT (XU XM, et al., 2017).

Tendo em vista esses fatores e relacionando com os estudos incluídos para síntese qualitativa nessa revisão, Ito M, et al. (2010) observaram os efeitos do Milnaciprano no tratamento da dor, observando os efeitos em relação ao aumento das doses durante 12 semanas inicialmente em $15 \mathrm{mg}$ e aumentada durante 0 tratamento para $100 \mathrm{mg}$ e também a condição psicológica do paciente, constatando que o medicamento possui efeito antinociceptivo (independendo se o paciente é depressivo ou não), propiciando o alívio da dor, sendo motivo de pauta os efeitos adversos com o aumento da dosagem.

Ainda, corroborando com esses achados, Kimura $\mathrm{H}$ et al. (2012) analisaram o mesmo fármaco durante $\mathrm{o}$ mesmo período de tempo e observaram os efeitos desse medicamento ao aumentar as dosagens durante 0 tratamento. Dessa forma, obtiveram que existe o efeito analgésico que o fármaco induz, porém, ao aumentar as doses, os efeitos adversos tendem a aumentar também, sendo avaliados esses efeitos e impostos que 
dentre os ADT, o Milnaciprano é o que apresenta índices mais baixos de efeitos demonstrados, em que a boca seca é o efeito adverso observado com maior frequência e de maneira sucinta no uso do mesmo, tornando o fármaco então viável para o tratamento de SDM.

Por outro lado, Nagashima W, et al. (2012) analisaram o efeito da Duloxetina da dor orofacial não orgânica. A Duloxetina foi administrada em uma dose flexível fixa de 20 a $40 \mathrm{mg} / \mathrm{d}$ a 41 pacientes com síndrome da boca ardente e/ou odontalgia atípica. A dor foi avaliada usando a escala visual analógica (EVA), sendo 12 semanas de tratamento. Foram analisados os dados de 29 pacientes que completaram o estudo. $O$ escore EVA às 12 semanas de tratamento foi significativamente menor do que o valor basal.

O tempo decorrido das pontuações da EVA revelou sua diminuição significativa a partir de 2 semanas de tratamento em comparação com a pontuação basal. Para investigar a influência dos sintomas depressivos basais no efeito analgésico da Duloxetina, os indivíduos foram divididos em 2 grupos com base na pontuação da Hamilton Depression Rating Scale na consulta inicial: grupos com $(\geq 8)$ e sem $(\leq 7)$ sintomas depressivos. Em contrapartida, comparado com outros fármacos como visto no estudo Hauser W, et al. (2010), como Venlafaxina, Milnaciprano e Amitriptilina, o mesmo apresenta efeitos menos potentes em decorrência de sua duração no organismo ser menor, não sendo, assim, o fármaco a primeira escolha para o devido tratamento.

Desse modo, Agius AM, et al. (2013) realizaram dois estudos avaliando os efeitos da baixa dose de Amitriptilina com Pindolol no tratamento de dor facial crônica por tensão, respectivamente um estudo observa a eficácia das baixas doses de AMT de forma isolada comparada com a junção AMT e Pindolol e o outro estudo observa as propriedades do fármaco analisando níveis séricos da serotonina no sangue.

No primeiro estudo, a partir da randomização dos pacientes em grupos ((A) AMT isolada e (B) AMT com Pindolol) durante 8 semanas, obtém-se como resultados, nas 3 primeiras semanas, que ambos os grupos apresentaram ação rápida e redução da dor, durante a continuação do tratamento o grupo $A$ apresenta efeitos positivos superiores devido a ação da droga funcionar de maneira periférica. Já no grupo $B$, observa-se também resultados positivos, porém inferiores, pois o Pindolol não acelera o processo de ação da AMT, pois o mesmo tem ação contrária (central).

Contudo, este fármaco apresenta resultados positivos na junção sinápticas, devido sua capacidade de bloquear receptores pré-sinápticos como o 5-HT1B reduzindo a dor, porém, não sendo esta propriedade o suficiente para obter melhores resultados, sendo a AMT utilizada de maneira isolada a terapia mais indicada.

No segundo estudo, a avaliação do grupo AMT com Pindolol (A) apresenta em exames sanguíneos a redução significativa da serotonina comparado a redução dos níveis do grupo placebo $(B)$, obtendo esses resultados a partir da possibilidade do tratamento combinado com dois fármacos no grupo $\mathrm{A}$.

Os mesmos reduzem a recaptação da serotonina sináptica do sistema nervoso central que é possibilitada devido a ação do Pindolol, que inibe o transportador de serotonina e aumenta os níveis de serotonina no sistema nervoso central, consecutivamente a esta ação são reduzidos os níveis de serotonina do sistema nervoso periférico, sendo então a serotonina absorvida mais facilmente pelas plaquetas e metabolizada no sangue. Obtendo então resultados que repercutem de maneira positiva, pois com a persistência da serotonina no sangue vai existir o aumento de sinapses que atenuam na dor, diminuindo-a.

Os dois artigos abordam o uso da AMT e a análise desses efeitos em conjunto com outro fármaco, assim como de maneira individualizada. Em decorrência disso, obtém a partir dos mesmos que os resultados vão ser variados e baseados na linha de pesquisa que está sendo analisada. Porém, considera-se que a AMT, segundo Micó JÁ, et al. (2010), por ser o padrão-ouro no tratamento com ADT para SDM apresenta taxas de sucesso em grande incidência.

No entanto, no primeiro artigo ao ser adicionada ao Pindolol, a mesma apresenta resultados positivos que quando comparados a utilização do ADT isolado, observando que não se tem a mesma rapidez de resposta a dor. Por outro lado, no segundo artigo, em que se muda a perspectiva da observação do estudo, a junção dos dois fármacos apresenta efeitos promissores na redução da sensação dolorosa, devido as propriedades do Pindolol observada com maior precisão na análise sanguínea. 
Ademais, Agius AM, et al. (2014) por meio de um estudo coorte acompanharam 240 pacientes durante 3 anos em uma clínica otorrinolaringológica. Os tipos de dor facial foram classificados de acordo com os critérios da Classificação Internacional de Dor de Cabeça. O índice de massa corporal, ocupação e nível educacional dos pacientes foram comparados com a população geral.

Os pacientes foram divididos em grupos pela sua condição: pacientes com dor no meio da face, pacientes tratados apenas com opioides, pacientes com convulsões crônicas, dentre outros. Dor no meio da face do tipo tensão e enxaqueca facial sem aura foram os tipos mais comuns de dor facial crônica. Decorridos os 3 anos, foi-se obtido que metade desses pacientes apresentaram melhoras relevantes sobre o tratamento e que neste decorrer de tempo aprenderam a usar de maneira responsável os fármacos. Os pacientes tratados com ADT (AMT por 8 semanas) estavam na porcentagem de pacientes livres de sensações dolorosas devido a atuação do fármaco de inibir a recaptação da serotonina-norepinefrina.

Não obstante, Alencar F, et al. (2014) analisaram o efeito de Ciclobenzaprina (CIC) e da Tizanidina (TZA) por meio de um programa educacional de autocuidado a pacientes com dor miofascial. Neste estudo, os pacientes foram randomizados e divididos em grupos que utilizavam CIC $10 \mathrm{mg}$; TZA 4mg e um grupo placebo obtiveram que os resultados de ambos durante o período foram iguais sem respostas significativas, realizando ressalvas para estudos futuros, a primeira ressalva remete ao aprofundamento do estudo sobre a TZA em relação a uma possível função que trará mais benefícios se a mesma for utilizada como pré-medicação, agindo de maneira preventiva.

A segunda ressalva remete a funcionalidade da TZA como uma espécie de medicamento "reserva" quando os pacientes não toleram bem a $\mathrm{CIC} \mathrm{e,} \mathrm{por} \mathrm{fim,} \mathrm{que} \mathrm{futuramente} \mathrm{as} \mathrm{pesquisas} \mathrm{realizadas} \mathrm{aumentem} \mathrm{as} \mathrm{doses}$ destes fármacos, afim de buscar os efeitos esperados.

Paradoxalmente, Haviv Y, et al. (2015) avaliaram o efeito da AMT e de um anticonvulsivante (Gabapentina) que são fármacos fortes que apresentam em quase $50 \%$ da população que os toma efeitos inibitórios da dor. Realizando a combinação de tratamento com esses dois fármacos existe a possibilidade da resolução de alguns problemas específicos.

Este estudo foi realizado com 42 pacientes e para aqueles que não apresentavam respostas com o uso da AMT eram encaminhados para o tratamento com Gabapentina, sendo os resultados medidos a partir da escala verbal de dor (EVD). Destes, 23 tiveram respostas satisfatórias a AMT, enquanto 19 fizeram uso apenas da Gabapentina.

Consequentemente, o grupo do anticonvulsivante apresentou resultados bem mais significativos devido ao fato da Gabapentina ter como mecanismo de ação o bloqueio dos canais de cálcio tipo L, que lançam o neurotransmissor modular e inibem a liberação de glutamato na medula espinhal, inibindo consecutivamente a dor.

Por conseguinte, Haviv $Y$, et al. (2019) trouxeram a mesma linha de estudo, mas agora analisando dois ADTs: Amitriptilina (AMP) e Nortriptilina (NOR). 50 pacientes foram divididos, em que 30 tomaram AMP e 20 NOR. Nos dois grupos, a média do desvio padrão do escore EVD no final do tratamento foi entre 2,92 a 3,2, sendo, assim, menor em comparação à linha de base $(6,4 \pm 1,75)$ e, portanto, considerada uma diferença clinicamente significativa.

Os escores iniciais foram semelhantes nos grupos AMT e NOR $(6,27 \pm 1,92$ e 6,78 $\pm 1,98)$. Os pacientes com NOR relataram um EVD final menor em comparação aos pacientes com AMT $(2,83 \pm 3,06$ vs 4,55 \pm 2,92). A taxa de melhora de $50 \%$ com o tratamento NOR foi melhor do que com o tratamento com AMT. Com isso, constatou-se que os dois medicamentos possuem alta capacidade de tratamento da dor, porém, a NOR por apresentar uma agressividade menor pode ser utilizada em doses mais altas sem causar tantos efeitos adversos aos pacientes.

Por outro lado, Kobayashi Y, et al. (2017) examinaram os efeitos da Duloxetina em pacientes com síndrome da boca ardente, que é uma dor não-orgânica na região orofacial. Foram administrados Duloxetina em 77 pacientes diagnosticados como portadores de síndrome da boca ardente ou odontalgia atípica por 12 semanas. 
A dose inicial de Duloxetina foi estabelecida em $20 \mathrm{mg} / \mathrm{d}$ e aumentada para $40 \mathrm{mg} / \mathrm{d}$ após uma semana. Com isso, foi realizada uma comparação dos níveis da EVA no início do tratamento com o término, observando uma redução com o uso da Duloxetina, sendo a dor agora caracterizada pelos pacientes como algo passageiro. No que diz respeito a concentrações plasmáticas, o fármaco não apresentou nenhuma relação com analgesia da Duloxetina, sendo observado então o motivo do mesmo não apresentar efeitos notórios.

Paradoxalmente a esses achados, Vase L, et al. (2016) em um estudo observando as taxas de concentrações do Milnaciprano em comparação com a Duloxetina, observaram que há presença de janela terapêutica no Milnaciprano entre a concentração plasmática e os seus efeitos analgésicos, enquanto que na Duloxetina não são observados os mesmos efeitos. Estes efeitos são descritos por Atkison JH, et al. (2011), que corroboram com estudo anterior afirmando que a resposta a terapia medicamentosa (analgesia) do Milnaciprano e concentração plasmática advém de o medicamento possuir farmacocinética linear, enquanto que na Duloxetina não existe o mesmo mecanismo.

De um modo geral, diversos fármacos podem desencadear efeitos positivos no tratamento de disfunções musculares faciais crônicas, destacando-se os antidepressivos tricíclicos entre os estudos devido a sua ampla aplicabilidade e resultados bem elucidados na literatura. Entretanto, devem ser pautados seus efeitos colaterais para condicionar o uso de forma moderada.

\section{CONSIDERAÇÕES FINAIS}

Em suma, os artigos observaram que os fármacos anti-inflamatórios não esteroidais (AINES), benzodiazepínico, tiazolidinedionas e antidepressivos possuem a capacidade de tratar com eficácia as dores miofasciais, principalmente dores crônicas, além de bruxismo e distúrbio do sono, devido a capacidade de possuir efeitos analgésicos. Porém, os antidepressivos tricíclicos (ADTs) são os mais utilizados, pois fazem a disponibilização de norepinefrina e serotonina nas junções sinápticas do sistema nervoso central. A Amitriptilina se destacou no estudo por ser considerada padrão-ouro para estes tratamentos. No entanto, necessitam-se de mais estudos para elucidar a aplicabilidade clínica desses medicamentos.

\section{AGRADECIMENTOS}

Agradecimento ao Centro Universitário Católica de Quixadá.

\section{REFERÊNCIAS}

1. ABBOUD WA, et al. Localized myofascial pain responds better than referring myofascial pain to botulinum toxin injections. International Journal Of Oral And Maxillofacial Surgery,2017; 46: 1417-1423.

2. AGIUS AM, et al. A Randomized Controlled Trial comparing the efficacy of low-dose amitriptyline, amitriptyline with pindolol and surrogate placebo in the treatment of chronic tension-type facial pain. Rhinology, 2013; 51: 143-153.

3. AGIUS AM, et al. Prospective three-year follow up of a cohort study of 240 patients with chronic facial pain. The Journal Of Laryngology \& Otology, 2014; 128: 518-526.

4. AGIUS AM, et al. Serial blood serotonin levels in a randomized controlled trial comparing the efficacy of low-dose amitriptyline, amitriptyline with pindolol and surrogate placebo in patients with chronic tension-type facial pain. Rhinology, 2013; 51: 236-242.

5. ALENCAR F, et al. Patient Education and Self-Care for the Management of Jaw Pain upon Awakening: a randomized controlled clinical trial comparing the effectiveness of adding pharmacologic treatment with cyclobenzaprine or tizanidine. Journal Of Oral \& Facial Pain And Headache, 2014; 28: 119-127.

6. ALVES ACM, et al. Utilização da terapia manual em paciente com disfunção temporomandibular - relato de caso. Revista Extensão e Sociedade da UFRN, 2012; 1: 108-118.

7. ATKINSON JH, et al. Is there a therapeutic window with some antidepressants for analgesic response? Curr Pain Headach Rep, 2011; 13: 93-99.

8. BATISTA SJ, et al. Tratamento fisioterapêutico na síndrome da dor miofascial e fibromialgia. Revista Dor, 2012; 13: 170-174.

9. BOAFO A, et al. Medications for sleep disturbance in children and adolescents with depression: a survey of Canadian child and adolescent psychiatrists. Child Adolesc Psychiatry Ment Health, 2020; 14: 10-17.

10. BROWN S, et al. A randomized controlled trial of amitriptyline versus gabapentin for complex regional pain syndrome type I and neuropathic pain in children. Scandinavian Journal Of Pain, 2016; 13: 156-163. 
11. CONNELLY ST, et al. Clinical outcomes of Botox injections for chronic temporomandibular disorders: do we understand how botox works on muscle, pain, and the brain? International Journal Of Oral And Maxillofacial Surgery, 2017; 46: 322-327.

12. DALEWSKI B, et al. Comparison of Early Effectiveness of Three Different Intervention Methods in Patients with Chronic Orofacial Pain: a randomized, controlled clinical trial.: A Randomized, Controlled Clinical Trial. Pain Research And Management, 2019; 2019: 1-9.

13. GARROTE CEM, et al. Antidepressivos em odontologia: indicações e cuidados. Revista Associação Paulista Cirugião-dentista, 2010; 64: 294-295.

14. HAUSER W, et al. Comparative efficacy and harms of duloxetine, milnacipran, and pregalin in fibromyalgia syndrome. J. Pain, 2010; 11: .505-521.

15. HAVIV Y, et al. Myofascial Pain: an open study on the pharmacotherapeutic response to stepped treatment with tricyclic antidepressants and gabapentin. Journal Of Oral \& Facial Pain And Headache, 2015; 29: 144-151.

16. HAVIV Y, et al. Nortriptyline Compared to Amitriptyline for the Treatment of Persistent Masticatory Myofascial Pain. Journal Of Oral \& Facial Pain And Headache, 2019; 33: 7-13.

17. ITO M, et al. Effectiveness of Milnacipran for the Treatment of Chronic Pain in the Orofacial Region. Clinical Neuropharmacology, 2010; 33: 79-83.

18. KIMURA H, et al. Plasma levels of milnacipran and its effectiveness for the treatment of chronic pain in the orofacial region. Human Psychopharmacology: Clinical and Experimental, 2012; 27: 322-328.

19. KOBAYASHI Y, et al. Duloxetine Plasma Concentrations and Its Effectiveness in the Treatment of Nonorganic Chronic Pain in the Orofacial Region. Clinical Neuropharmacology, 2017; 40: 163-168.

20. KRONER JW, et al. Trajectory of Improvement in Children and Adolescents With Chronic Migraine: results from the cognitive-behavioral therapy and amitriptyline trial. The Journal Of Pain, 2017; 18: 637-644.

21. LYONS DN, et al. Combination Drug Therapy of Pioglitazone and D-cycloserine Attenuates Chronic Orofacial Neuropathic Pain and Anxiety by Improving Mitochondrial Function Following Trigeminal Nerve Injury. Clin. J. Pain, 2018; 34: 168-177.

22. MICO JA, et al. Antidepressives and pain. Trends in Pharmacological Sciences, 2010; 27: 348-354.

23. NAGAKURA Y, et al. Spontaneous pain-associated facial expression and efficacy of clinically used drugs in the reserpine-induced rat model of fibromyalgia. Eur. J. Pharmacol., 2019; 864: 172-716.

24. NAGASHIMA W, et al. Effectiveness of Duloxetine for the Treatment of Chronic Nonorganic Orofacial Pain. Clinical Neuropharmacology, 2012; 35: 273-277.

25. URQUHART DM, et al. Efficacy of Low-Dose Amitriptyline for Chronic Low Back Pain. Jama Internal Medicine, 2018; 178: 1474-1481.

26. VAROLI FK, et al. Analgesia Evaluation of 2 NSAID Drugs as Adjuvant in Management of Chronic Temporomandibular Disorders. The Scientific World Journal, 2015; 2015: 1-7.

27. VASE L, et al. Placebo, Nocebo and neuropathic pain. Pain, 2016; 157: 98-105.

28. VON WA, et al. Selective serotonin reuptake inhibitors and tricyclic antidepressants in the acute treatment of chronic depression and dysthymia: a systematic review and meta-analysis. J Affect Disord, 2013;144: 7-15.

29. XU XM, et al. Tricyclic antidepressants for preventing migraine in adults. Medicine (Baltimore), 2017 ; 96: 6989-6995. 